\title{
Programa educativo para desarrollar competencias comunicativas en niños hospitalizados en el Instituto Nacional de Rehabilitación
}

\author{
Lic. Cinthya N. Gutiérrez-Huerta, ${ }^{*}$ Lic. Rocío Berrocal-García*
}

\section{RESUMEN}

Una enfermedad o la discapacidad de niños hospitalizados, les impide ir a la escuela por un tiempo prolongado o en forma permanente. Este problema ofreció una oportunidad de investigar y elaborar un programa educativo para desarrollar competencias comunicativas (comprensión lectora, expresión escrita y expresión oral), que contribuyan a la recuperación física, escolar y social del niño hospitalizado, a través de métodos y estrategias de la pedagogía hospitalaria.

Palabras clave: Pedagogía hospitalaria, competencias comunicativas, atención integral del paciente.

\section{ABSTRACT}

Illness or disability of hospitalized children, creates the impossibility to go to school for prolonged period of time or permanently. This situation offered an opportunity to study and to develop an educational program in order to enhance communication skills: reading comprehension, written expression and oral expression, which will contribute to intellectual improvement, through methods and strategies of hospital pedagogy.

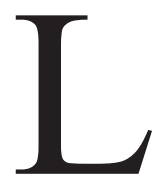

a acción educativa en el Instituto Nacional de Rehabilitación (INR), dio la oportunidad de identificar una serie de problemas en los procesos comunicativos de los niños hospitalizados: la comprensión lectora, la elaboración de mensajes por escrito y la comunicación interpersonal.

En base a lo anterior, se comprende la necesidad de elaborar un programa pedagógico para desarrollar competencias comunicativas, útiles a lo largo de la vida de los niños.

La elaboración del programa parte de la pregunta: ¿Qué necesita conocer, saber y hacer el niño hospitalizado para desarrollarse dentro de una sociedad que cada día demanda mayores conocimientos y habilidades de los individuos? Esto implica un abordaje distinto al proceso de enseñanza-

\footnotetext{
* Articulo extraído de la tesis "Programa educativo para desarrollar competencias comunicativas en niños hospitalizados, en el Instituto Nacional de Rehabilitación", para obtener el titulo de Licenciada en Pedagogía por la Universidad Pedagógica Nacional.

Correspondencia: Lic. Cinthya N. Gutiérrez-Huerta. $3^{a}$. Cerrada de Potrero M 50 L11. Col. San Bartolo Ameyalco. Del. Álvaro Obregón Cp. 01800 . Tel. 5515719655 . Correo electrónico: cinn_gz_huerta@hotmail.com
}

Este artículo debe citarse: Gutiérrez-Huerta CN, Berrocal-García R. Programa educativo para desarrollar competencias comunicativas en niños hospitalizados en el Instituto Nacional de Rehabilitación. Acta Pediatr Mex 2010;31(1):16-21.

Recibido: octubre, 2009. Aceptado: diciembre, 2009. aprendizaje, no el de la enseñanza tradicional, sino de la que responda a las nuevas necesidades educativas, para propiciar el desarrollo de competencias comunicativas; que retome los conocimientos previos de los niños y el contexto hospitalario en el que se encuentran, es decir, guiar este proceso con base en un enfoque constructivo, fundado en los cuatro pilares de la educación del informe Delors.

\section{EDUCACIÓN PARA LA VIDA}

Según el Informe Delors a la UNESCO, la educación debe enseñar a vivir mejor mediante el conocimiento, y tiene la misión de permitir en todos, sin excepción, hacer fructificar los talentos y todas sus capacidades de creación ${ }^{1}$.

Para cumplir esta tarea, la educación se estructura con base en cuatro aprendizajes fundamentales, que para cada persona, en el transcurso de la vida, serán los pilares de la formación de una cultura personal: aprender a conocer, aprender a hacer, aprender a vivir juntos y aprender a ser.

\section{ORÍGENES DE LA PEDAGOGÍA HOSPITALARIA}

La pedagogía terapéutica fue la primera en articular la atención médica con la educativa; posteriormente, en la década de 1970 este término evolucionó al de educación 
especial, que se amplía y diversifica y hace referencia a las ayudas especiales para apoyar a todo niño que lo necesite. A raíz de ésta, surge la educación inclusiva, que se extiende a todos los servicios de la educación, comprometida con el desarrollo de los niños ${ }^{2}$.

La pedagogía hospitalaria se inserta en la educación inclusiva (cuadro 1), desarrolla estrategias para favorecer la educación del niño hospitalizado, por medio de un proyecto educativo y la formación íntegra del pedagogo hospitalario.

Algunos de sus objetivos son:

a) beneficiar la recuperación del niño enfermo y

b) mejorar su calidad de vida durante su hospitalización.

Cuadro 1. Orígenes de la pedagogía hospitalaria. Fuente: GrauOrtiz, 2001.

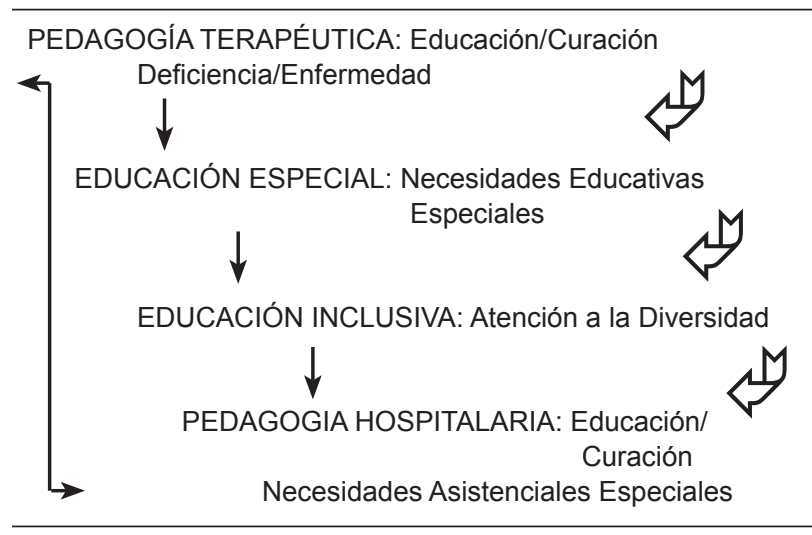

Para lograr éstos, la pedagogía hospitalaria, debe ser flexible en sus métodos y estrategias de enseñanzaaprendizaje con una cultura orientada a la innovación y adaptación de los recursos para el mejor desarrollo de los niños ${ }^{2}$.

\section{LAS COMPETENCIAS EN EDUCACIÓN}

La Secretaría de Educación Pública (SEP) define las competencias como "el conjunto de conocimientos, conceptos, percepciones, saberes, creencias, habilidades, destrezas, estrategias, procedimientos, valores y actitudes, para enfrentar los retos cotidianos, mejorar nuestra vida, comprender y dar sentido al mundo que nos rodea" ${ }^{3}$.

Esta definición retoma los cuatro pilares de la educación, del Informe Delors.

\section{DESCRIPCIÓN DE LA COMPETENCIA COMUNICA- TIVA: EXPRESIÓN ORAL, LECTURA Y EXPRESIÓN ESCRITA}

Niño Rojas menciona que la competencia comunicativa: "consiste en un saber especifico que representa la competencia lingüística y el saber aplicarlo es la competencia pragmática, que habilita a miembros de un grupo social para realizar actos comunicativos, según sus necesidades por determinados canales y códigos" 4 .

La lectura, la escritura y la expresión oral son actos comunicativos que se dan a lo largo de la vida, por lo cual deben ser desarrolladas y fortalecidas como competencias comunicativas, a fin de que el paciente comprenda su utilidad en la vida cotidiana.

La expresión oral, antes que la lectura y la escritura, es la base fundamental en la educación de los niños, pues durante su educación básica, se presenta en la convivencia con sus compañeros, en la relación con el profesor (socialización). Cuando un niño no sabe leer ni escribir, el conocimiento y las competencias fundamentales para la vida se desarrollan de forma oral. Como dice Ibáñez " $E l$ trabajo escolar de la comprensión y la expresión oral tiene la ventaja de poder ser tratado y sistematizado mucho antes de que el niño sepa leer y escribir" ${ }^{5}$.

Las actividades lúdicas y recreativas permiten que el niño se inicie en la competencia comunicativa oral, es decir, que se relacione y se comunique positivamente con otros niños y con los docentes, puesto que "uno de los fines que se persigue es el diálogo continuo con los niños a fin de que sin inhibiciones, se comuniquen con los adultos a través del lenguaje verbal" 5 .

Así mismo, la lectura y la escritura no se pueden enseñar de forma separada, "La lectura es una forma de escritura: cuando leo, le doy vida a lo que está escrito; luego los dos procesos son complementarios" " ${ }^{6}$. Es decir, el desarrollo de una implica necesariamente el desarrollo de la otra; si una no está presente en el desarrollo cognitivo del sujeto, la otra no puede desarrollarse; es como si el sujeto no pudiera leer la realidad, el contexto en el que crece.

Hurtado menciona que "Escribir es un proceso constructivo de representación de significados a través de los cuales se expresan los pensamientos y los sentimientos con una intención comunicativa" " ${ }^{7}$. Esto implica que los niños aprendan a comunicarse primero de forma oral, y desarrollen la capacidad argumentadora por medio de la 
convivencia con sus iguales y sus mayores; y posteriormente iniciarlos en el proceso de lecto-escritura. De esta forma, cuando el niño lee las instrucciones de un ejercicio escolar (trata de entender lo que dice un texto), y escribe un recado para algún familiar (escribe para comunicarse), sabe que por medio de la lectura y la escritura puede dar a conocer lo que piensa o necesita.

\section{"PROGRAMA EDUCATIVO PARA DESARROLLAR COMPETENCIAS COMUNICATIVAS EN NIÑOS HOSPI- TALIZADOS, EN EL INR"}

El programa educativo pretende que durante su hospitalización, los pacientes desarrollen y fortalezcan sus competencias comunicativas, por medio de una atención y educación especiales que, además, contrarresten en lo posible, los efectos derivados de su hospitalización y favorezcan el proceso de su reinserción escolar y social, que como dicen Grau y Ortiz "sería como trazar un puente o crear un atajo para facilitar su acceso a la educación con el propósito de que su deficiencia no se convierta en un obstáculo infranqueable que le impida llevar a cabo su proceso de formación, su itinerario evolutivo como persona" ${ }^{2}$.

En el programa se desarrollan competencias comunicativas desde la perspectiva de la comunicación endógena ${ }^{8}$, la construcción social de la mente y la Zona de Desarrollo Próximo $(Z D P)^{9}$, en el marco de una educación liberadora $^{10}$. Se consultaron los programas oficiales: Programa de Educación Preescolar (PEP), Competencias para la Educación Primaria en el Distrito Federal 2004-2005, que cuentan con el enfoque por competencias y el libro Desarrollo de competencias en educación básica: desde preescolar hasta secundaria.

La tesis fundamental sobre la que se diseña este programa es que las competencias comunicativas entendidas como la lectura de comprensión, la expresión escrita y la expresión oral, son la base de las relaciones escolares y sociales de todo individuo. Es decir, desarrollar la competencia comunicativa significa usar el conocimiento para la resolución de problemas de la vida cotidiana.

\section{Características de la Población y del Contexto}

El programa se aplicó en el Instituto Nacional de Rehabilitación (INR).

Para seleccionar a los pacientes se adoptaron los siguientes criterios:
- Estar hospitalizado en el área de Ortopedia Pediátrica del INR.

- Estar inscrito en alguna institución educativa a nivel primaria.

- Tener una edad de 6 a 11 años.

\section{Hipótesis de Trabajo}

- El paciente hospitalizado incrementa sus competencias comunicativas si se crean situaciones en la que puedan expresar pensamientos, necesidades y sentimientos mediante la lectura, la escritura y la expresión oral.

- Si la lectura, la escritura y la expresión oral se logran integrar en situaciones didácticas que sean significativas al alumno, se favorecerá su competencia comunicativa.

\section{Propósitos del Programa}

- Desarrollar simultáneamente la lectura, la escritura y la expresión oral como competencias comunicativas.

- Utilizar materiales accesibles y novedosos en situaciones didácticas.

- Promover el uso crítico de las Tecnologías de la Información y la Comunicación (TIC).

- Promover la comunicación entre el docente y el paciente en un ambiente de confianza para beneficiar el desarrollo de las competencias comunicativas.

- Posibilitar los procesos de relación y socialización del niño hospitalizado con otros compañeros.

\section{Estructura y Diseño}

La estructura y diseño del programa se realizó sobre cuatro ejes retomados del programa de Competencias para la educación primaria en el Distrito Federal, 2004-2005: 1) comprensión del medio natural, social y cultural; 2) actitudes y valores para la convivencia; 3 ) comunicación; 4) lógica-matemática que a su vez están divididos en diez temas que favorecen el desarrollo de las competencias comunicativas durante 20 sesiones. Las estrategias que se utilizaron en el programa se adecuaron según los requerimientos y características del tema y la población. Los instrumentos de evaluación son: cuestionario prediagnóstico de competencias comunicativas de $1^{\circ}$ a $3^{\circ} y$ de $4^{\circ}$ a $6^{\circ}$; cuestionarios post-diagnóstico, elaborados de acuerdo a cada uno de los 10 temas; instrumento para la 
evaluación del desarrollo de competencias comunicativas; $y$ criterios de evaluación para $1^{\circ}$ a $3^{\circ}$ y para $4^{\circ}$ a $6^{\circ}$.

El programa se compone por: eje (correspondiente al programa oficial); tema; competencia comunicativa (lectura de comprensión, expresión escrita y expresión oral); indicadores de desempeño, conocimientos, habilidades de pensamiento, destreza y actitud (elementos que componen la competencia); la situación didáctica; secuencia didáctica; duración de la sesión; competencias transversales; material a utilizar; y los mecanismos de evaluación. (Cuadro 2).

Cuadro 2. Estructura del programa

\section{Eje}

Tema

Competencia: Lectura, escritura y expresión oral.

Indicadores Conocimientos Habilidades Destreza Actitud de desempeño de pensamiento

Situación didáctica: Escenario de aprendizaje, el conjunto de actividades que articuladas entre sí promueven que el alumno desarrolle la competencia.

Secuencia didáctica de la $1^{a}$ y $2^{\circ}$ sesión: Orden en que deben estar las actividades de aprendizaje.

Duración: dos sesiones de tres horas cada una

Competencias transversales: Conocimientos generales a los que se recurre para apoyar el desarrollo de la competencia.

Materiales a utilizar:

Mecanismo de evaluación: Cuestionario pre-diagnóstico, evaluación por portafolios, indicadores de desempeño y cuestionario post-diagnóstico

\section{RESULTADOS}

\section{Características Demográficas de la Población}

En la figura 1 se observa en una barra blanca a los 20 niños y en una barra gris a las ocho niñas que participaron en el programa, lo que da un total de 28 pacientes.

El programa se aplicó formando dos grupos uno de $1^{\circ}$ a $3^{\circ}$ y otro de $4^{\circ}$ a $6^{\circ}$. El primero fue con 12 pacientes y el segundo con 16 .

La figura 2 es una de las 28 gráficas (una para cada niño) que muestra el desarrollo de las competencias comunicativas del paciente, antes y después de la aplicación del programa, y se identifica con los siguientes datos: folio (F) y nombre, entre paréntesis: edad-género-grado escolar.

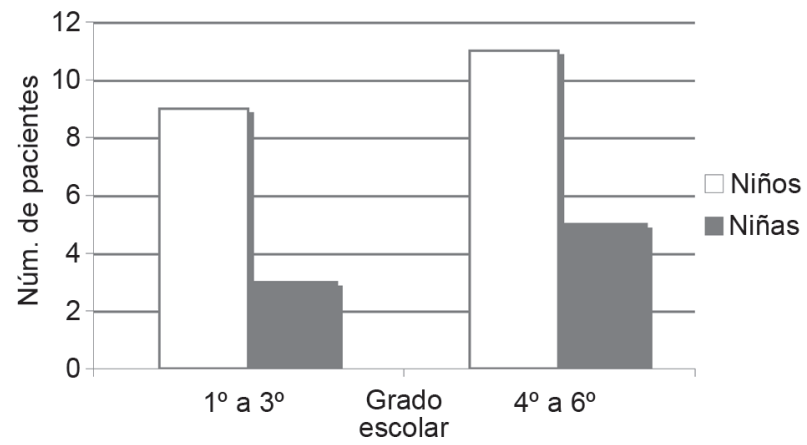

Figura 1. Características demográficas de la población. Distribución por grado y por género.

F 16 Daniel $\left(9-\mathrm{M}^{\circ} 4^{\circ}\right)$

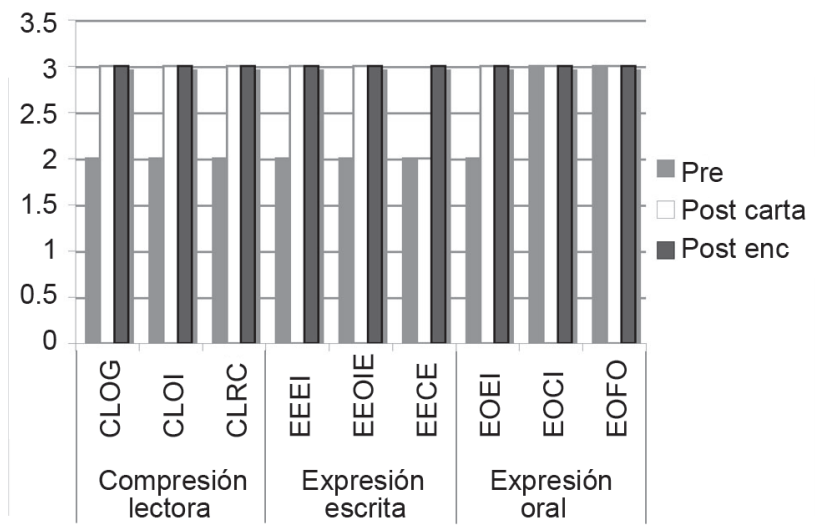

Figura 2. Desarrollo de las competencias comunicativas de Daniel. La leyenda Pre se identifica como pre-diagnóstico y Pos como postdiagnóstico. Para la sistematización de los datos. A la evaluación "bueno, regular y deficiente" se les asignaron valores númericos: 3 , 2,1 , respectivamente. En la parte inferior se encuentran las siglas de los elementos que conforman cada competencia. Comprensión lectora: comprensión global (CLCG); obtención de información (CLOI); reflexiona sobre el contenido (CLRC). Expresión escrita: expresión de ideas (EEEI); ordenamiento de ideas (EEOIE); corrección de la expresión (EECE). Expresión oral: expresión de ideas (EOEI); comunicación de sus ideas (EOCI); fluidez oral (EOFO).

En la gráfica de Daniel se observan dos temas, la carta y la encuesta, la primera barra representa el cuestionario pre-diagnóstico; la segunda, el post diagnóstico del tema la carta; y la tercera barra, el post diagnóstico del tema la encuesta.

Al inicio de la aplicación, la compresión lectora tuvo una primera evaluación de regular; en los temas y evaluaciones posteriores, se observa una notable mejoría a bueno en los tres aspectos, al obtener información, reflexionar sobre el contenido y comprender globalmente el tríptico informativo para realizar su encuesta. 
$\mathrm{Al}$ igual que en la lectura, la expresión escrita inicialmente fue regular, y mejoró a buena al organizar, clasificar y seleccionar la información de la encuesta para realizar e interpretar su gráfica.

La actividad propició que su expresión oral, permaneciera buena durante todas las sesiones, pues se fomentó la participación de los niños en todo momento. De esta manera Daniel formuló la pregunta de encuesta y comunicó con claridad sus ideas para que las comprendieran otros.

En este tema, los niños que participaron se mostraron entusiasmados al realizar sus encuestas y superaron el miedo al ambiente hospitalario, como Daniel que a pesar de su condición médica, desarrolló su zona de desarrollo próximo al realizar dos encuestas más por iniciativa propia.

\section{El Programa}

El programa educativo tuvo efectos benéficos en el paciente, aun sin un propósito terapéutico explícito.

Las situaciones didácticas promovieron la participación constante de los pacientes con una perspectiva crítica en cada tema. Ejemplo. F13 Juan (11-M-6 $\left.{ }^{\circ}\right)$, daba constancia de su interés por cambiar la situación de desigualdad de género. Comentó: "es muy difícil jugar con niños y niñas en la escuela, porque mis compañeros se van a burlar de mí, entonces lo que tengo que hacer es enseñarles el tríptico para que ellos sepan lo que es la igualdad de género."

El uso crítico de las TIC permitió identificar las situaciones de la vida cotidiana donde se cumplen o no los derechos de los niños, por ejemplo: ¿Cuál derecho de los niños es el que menos se cumple? El paciente, F 9 Pablo (6-M-1 $\left.{ }^{\circ}\right)$ contestó: "De la explotación, porque hay niños trabajando en la calle y la educación porque no todos los niños estudian".

\section{El Paciente}

Los pacientes se comunicaron y convivieron entre sí mediante cartas y fue muy significativo para ellos saber que no eran los únicos en sentir temor por el ambiente hospitalario en el que se encontraban. Ejemplo: F14 Gerardo (8-M-3 ${ }^{\circ}$ ) escribió “iHola Daniel! ¿Cómo te sientes? Espero que te recuperes pronto, a mi me van a operar hoy, estoy nervioso porque no me gusta que me piquen."

Desarrolló sus competencias comunicativas, identificó la utilidad de sus conocimientos y los medios de comuni- cación para la aplicación de las competencias en su vida cotidiana, y transformó, descubrió y reelaboró su conocimiento junto con el docente y otros pacientes.

\section{REFLEXIONES FINALES}

El Programa logró hacer accesible la educación a niños hospitalizados; se cumplió el propósito universal de la educación para todos.

Se logró un trabajo conjunto entre el personal de salud, los familiares y el personal educativo, tarea que se creía muy difícil.

El ambiente hospitalario puede parecer agresivo para los niños, por lo que el aprendizaje para ellos se convirtió en un logro.

Los resultados obtenidos de la investigación reflejan la necesidad de contar con este programa educativo en un hospital, con contenidos extraídos del entorno de los niños, que realmente sean significativos para ellos, y así los puedan utilizar en otros momentos.

El profesional de la educación, muchas veces no se concede la oportunidad de abrir nuevos caminos en el campo laboral, es decir, se concentra en la labor docente o de capacitación y deja de lado el desarrollo intelectual de la población infantil hospitalizada o enferma a través de diseños curriculares acordes a ésta. Sin embargo, este trabajo es una prueba de cómo podemos adentrarnos en un ámbito poco explorado: La pedagogía hospitalaria.

Las necesidades médicas y educativas de una población vulnerable, convocan a los profesionales de la salud y la educación, a conjuntar esfuerzos para la recuperación íntegra de los niños enfermos, dentro o fuera del hospital.

El programa educativo propuesto implica comprometerse formal y concretamente con la población infantil hospitalizada. Requerirá para su aplicación en otros institutos de salud o centros educativos, adecuaciones para que pueda ser de utilidad en la práctica educativa y el proceso de enseñanza-aprendizaje en una población vulnerable.

El niño hospitalizado que estudia, no pierde el año escolar. Aprende a adaptarse a su entorno. Desarrolla su potencial intelectual.

\section{REFERENCIAS}

1. Delors J. La educación encierra un tesoro. México, D. F.: ed. Correo de la UNESCO; 1996. p. 9-19. 
2. Grau-Rubio C, y Ortiz C. La Pedagogía Hospitalaria en el marco de una educación inclusiva. Málaga, España: Ed. Aljibe; 2001. p. 23-34.

3. SEP. Competencias para la educación primaria en el Distrito Federal, 2004-2005. México, D. F.: ed. SEP; 200. p.3.

4. Niño-Rojas VM. Competencias en la comunicación. Colombia: ed. ECOE; 2003: p. 27.

5. Ibáñez-Sandín C. El proyecto de educación infantil y su práctica en el aula. Madrid, España: ed. Muralla; 1994. p. 168-170.

6. Montealegre A. Juegos comunicativos: estrategias para desarrollar la lectoescritura. Bogotá, Colombia: ed. Magisterio; 1995. p. 43.

7. Díaz-H LE y Echeberry-Z CE. Enseñar, aprender, leer y escribir. Bogotá, Colombia: ed. Magisterio. 1998. p. 3.

8. Kaplún M. Una pedagogía de la comunicación. Madrid, España: ed. De la Torre; 1998. p. 252.

9. Wertsch JV. Vigotsky y la formación social de la mente. Cognición y desarrollo humano. España: ed. Paidos; 1995. p. 264.

10. Freire $\mathrm{P}$ y Donaldo M. Alfabetización. Lectura de la palabra y lectura de la realidad. Barcelona, España: ed. Paidos; 1989. p. 176.

11. Frade Rubio L. Desarrollo de competencias en educación básica: desde preescolar hasta secundaria. México, D. F.: ed. Calidad educativa consultores; 2007. p. 140.
12. Freire P. Pedagogía del oprimido. Uruguay: ed. Siglo XXI; 1970. p. 245.

13. Blanck, G, Dubrovsky S. Vigotsky: su proyección en el pensamiento actual. Buenos Aires, Argentina: ed. Novedades Educativas; 2000. p. 95.

14. Freire P. La importancia del acto de leer. Caracas, Venezuela: ed. Laboratorio Educativo; 2006. p. 101.

15. Freire P. La educación como práctica de la libertad. México, D. F.: ed. Siglo XXI; 2005. p. 151.

16. Castorina JA, Dubrobsky S. Psicología, cultura y educación. Perspectivas de la obra de Vigotsky. Argentina: ed. Noveduc; 2004. p. 143.

17. Dubrobsky S. Vigotsky: su proyección en el pensamiento actual. Argentina: ed. Noveduc; 2000. p. 95.

18. Moll LC. Vigotsky y la educación: connotaciones y aplicaciones de la psicología sociohistórica en la educación. Buenos Aires, Argentina: ed. Aique; 1993. p. 493.

19. Freire P. Alfabetización: lectura de la palabra y lectura de la realidad. México, D. F.: ed. Paidós; 1989. p. 176.

20. Freire P. Cartas a quien pretende enseñar. México, D. F.: ed. Siglo XXI; 1994. p. 141.

21. García-González E. Vigotsky: la construcción histórica de la psique. México, D. F.: ed. Biblioteca grandes educadores; 2000. p. 147.

22. SEP. Programa de Educación Preescolar 2004 